\title{
Premiers résultats de l'étude MELS (Décisions médicales en fin de vie en Suisse)
}

Les décisions médicales en fin de vie font l'objet des appréciations les plus diverses, non seulement dans l'ensemble de la population, mais également au sein du corps médical. C'est pourquoi, une réflexion approfondie sur l'approche de l'agonie et de la mort elle-même s'impose. Les nombreuses émissions diffusées sur ce sujet par les médias en témoignent. L'actualité de ces questions et leur importance au niveau politique ne sont pas seulement les signes d'une évolution, mais révèlent également la nécessité d'instaurer une réglementation. A cet égard, la participation de personnes confrontées à ces problèmes dans leur activité quotidienne est indispensable. Les résultats de l'étude MELS sur la fréquence des différentes formes d'assistance au décès, présentés dans cette revue (pages 1730-1732; paru en original dans Lancet 2003;362:345-50), mais également l'évaluation encore en cours de la deuxième partie de l'étude portant sur les attitudes, les expériences et les opinions des médecins face aux mourants, y contribuent de façon conséquente.
L'Académie Suisse des Sciences Médicales (ASSM) se préoccupe depuis longtemps du problème des décisions médicales en fin de vie. Déjà en 1976 elle avait élaboré des directives médicoéthiques sur ce thème; celles-ci sont actuellement - et une fois de plus - en cours de révision. Les résultats de l'étude MELS démontrent en particulier que, pour environ la moitié de tous les décès (ou deux tiers des décès prévus) en Suisse, des décisions médicales interviennent. Grâce à ces résultats, il existe enfin des données indispensables à une réflexion objective sur ce thème.

Grâce aux nombreuses réponses (deux tiers des questionnaires ont été retournés), des conclusions pertinentes - pas toujours faciles à interpréter - ont pu être dégagées. Elles témoignent en tout cas de l'importance que le corps médical a accordé à ce sondage. L'Académie Suisse des Sciences Médicales (ASSM) remercie chaleureusement tous ceux qui ont pris le temps de remplir ces formulaires.

Pr Werner Stauffacher, président de l'ASSM 